\title{
Implicancia del Decreto 117 en la demanda sísmica y respuesta global en edificios de Antofagasta
}

\section{Implication of the Act 117 in the seismic demand and global response in buildings of Antofagasta}

\section{Juan Music, Patricio Tapia y Wagner Fleming}

Fecha de entrega: 21 de octubre 2011

Fecha de aceptación: 19 de abril 2012

Departamento de Ingeniería Civil, Universidad Católica del Norte, Avda. Angamos 0610, Antofagasta, Chile, jmusic@ucn.cl, ptapia@ucn.cl,wfleming@ucn.cl

Este artículo resume los resultados del estudio realizado sobre las implicancias del Decreto 117 del Ministerio de Vivienda y Urbanismo publicado en Febrero de 2011, en la demanda sísmica y respuesta global en edificios altos (mayor o igual a 10 pisos) ubicados en la ciudad de Antofagasta. Se describe en primer lugar las características geotécnicas de los suelos y se presenta un catastro de los edificios existentes en Antofagasta. Aun cuando en la ciudad se puede encontrar los tres tipos principales de suelos que identifica el decreto, para los cuales se define un espectro de diseño, la mayoría de los edificios altos se encuentran fundados en roca. Posteriormente se seleccionan nueve edificios, a los que se les realizó un análisis modal espectral según la norma NCh433 Of. 96 Mod. 2009 y según lo dispuesto por el Decreto 117. Se comparan los resultados obtenidos por ambas disposiciones en términos de esfuerzo de corte y momento volcante basal y esfuerzo de corte a nivel de piso. Finamente se concluye que la respuesta global de edificios fundados en roca (Tipo I) es muy similar aplicando ambas normativas. Por otra parte, para suelo Tipo II, se aprecian notorias diferencias de momentos volcantes y desplazamientos superiores y máximos de entre piso.

Palabras clave: normativa sísmica, demanda sísmica, respuesta sísmica, momento volcante, desplazamiento
In this paper the results of the study about the effect of the changes in the seismic code included in the Act 117 approved by the Ministry of Housing and Urbanism in February 2011 are summarised. The discussion is focused on the demand and response of high-rise buildings (ten or more floors) located in Antofagasta. First, a geotechnical description of the soil conditions and information of buildings are presented. Even though the three main soil types described in the Act can be found as foundation soils in the city, and each one has a defined design spectrum, most of the high-rise buildings are founded on rock. From the registered information, nine tall buildings were selected for modal spectral analysis under both the NCh433 Of. 96 Mod. 2009 and the Act 117. Results obtained using both regulations are compared in terms of shear force and overturning moment at the base level and shear force at different floor levels. Finally, on one hand it is concluded that the seismic response of buildings founded on rock (type I) is very similar considering both regulations. On the other hand, for soil type II, significant differences were found in the overturning moments and upper displacements and maximum relative displacement between floors.

Keywords: seismic code, seismic demand, seismic response, overturning moments, displacement

\section{Introducción}

Producto del terremoto de magnitud momento 8.8 ocurrido en el centro y sur de Chile el 27 de febrero del año 2010, específicamente entre las regiones de Valparaíso y Bío Bío, el Ministerio de Vivienda y Urbanismo MINVU conformó un panel de expertos en materias relativas al diseño sísmico de edificios. Este comité estudió las modificaciones necesarias tanto para el código sísmico como para la normativa de diseño de hormigón armado, recogiendo la experiencia de los daños observados en edificios en la zona centro y sur del país. Como resultado de este trabajo en febrero del 2011 se dictaron dos decretos. Por un lado se promulgó el Decreto 117 (MINVU, 2011a), que fija el 
diseño sísmico de edificios, modificando fundamentalmente la clasificación sísmica de suelos de fundación y el espectro de diseño que hasta la fecha era regulado por la norma NCh433 of 96 Mod. 2009 (INN, 2010). Si bien en el decreto se definen cinco tipos de suelo, se explicitan los espectros de diseño para suelos Tipo I, II y III. Por otro lado se promulgó el Decreto 118 (MINVU, 2011b), que fija los requisitos de diseño y cálculo para estructuras de hormigón armado. Usando edificios de la zona central, se han realizado una serie de estudios comparativos de la respuesta de estos edificios frente a la nueva demanda sísmica dada por el espectro del Decreto 117 y las implicancias del Decreto 118 en el requerimiento de refuerzo estructural. El presente trabajo se enfoca en analizar las implicancias del Decreto 117 en los edificios altos ubicados en la ciudad de Antofagasta. Para ello se seleccionan nueve edificios a los cuales se les realiza un análisis modal espectral según la norma NCh433 Of 96 Mod. 2009 y según lo dispuesto por el Decreto 117. Producto de ello, se comparan los resultados obtenidos por ambas disposiciones, tanto a nivel de esfuerzo de corte y momento volcante basal, como de esfuerzo de corte a nivel de piso.

En diciembre del 2011 apareció el decreto 61 (MINVU 2011c), el cual deroga el decreto 117 considerado en este trabajo, escrito antes de su derogación. Sin embargo, tanto en el caso de roca como de suelo denso (Tipo I y II), ambos decretos coinciden medularmente en la clasificación sísmica del material de fundación. Aunque los espectros propuestos son algo diferentes, este trabajo justamente entrega posibles razones de esa diferencia.

\section{Características geotécnicas del suelo de fundación de Antofagasta}

El daño final generado por un terremoto depende fundamentalmente de la amenaza sísmica y de la vulnerabilidad de las estructuras. Es esperable que estructuras vulnerables presenten daño cuando ellas están ubicadas en zonas donde la sismicidad es alta. La demanda sísmica en una cierta zona depende de los parámetros del sismo como la magnitud y la distancia epicentral y de las condiciones del sitio. En este aspecto, el suelo de fundación juega un rol muy importante a la hora de estimar la respuesta de las estructuras ante un evento sísmico de proporciones. De acuerdo a su rigidez, resistencia al corte y grado de compactación, los suelos se pueden considerar como adecuados o no para soportar las estructuras. Debido a su característica de no amplificar la amplitud de las ondas sísmicas, los suelos tipo roca son los más adecuados para la fundación de infraestructura. Cualquier otro tipo de suelos, en función de su espesor, densidad y rigidez, producirá un efecto local de amplificación de ondas que redundará en un mayor desplazamiento del suelo, comparado con el suelo tipo roca. El Decreto 117 del Ministerio de Vivienda y Urbanismo establece una nueva forma de caracterización sísmica de los suelos de fundación, donde destaca la obligatoriedad de determinar la Velocidad de Propagación de Ondas de Corte $\left(\mathrm{v}_{\mathrm{s}}\right)$ para todos los tipos de suelo, especificando los umbrales respectivos. El decreto también estipula la realización de otros ensayos como el RQD (Rock Quality Designation) para el caso de roca y SPT (Standard Penetration Test) para suelos. Si bien es cierto estos ensayos estaban considerados en la anterior normativa, junto con los ensayos de Densidad Relativa y Proctor Modificado, el decreto descarta estos últimos y establece que la caracterización debe basarse fundamentalmente en el valor de $\mathrm{v}_{\mathrm{s}}$. El decreto entrega una clasificación sísmica de suelos basado en cinco tipos (uno más que lo estipulado por la NCh433 Of. 96 Mod. 2009), sin embargo el suelo tipo $\mathrm{V}$ corresponde a suelos de muy mala calidad que deben ser especial y cuidadosamente estudiados. Para el caso de la caracterización de la roca, se deben utilizar al menos dos ensayos, teniendo a la Velocidad de Ondas de Corte como obligatorio y a los ensayos RQD y Compresión no confinada como adicionales. La clasificación sísmica de suelos tipo II, III y IV se debe justificar mediante valores de la Velocidad de Onda de Corte más valores de SPT (para arena) y compresión no confinada (para arcilla).

La ciudad de Antofagasta está fundada en su mayor parte en roca, lo que de alguna forma la ha protegido frente a los embates de grandes sismos. En el año 1994 se realizó un estudio geotécnico de la ciudad (Aguilera, 1994), basado en prospecciones de profundidades no mayores a $3 \mathrm{~m}$, más otros antecedentes de sondajes y estudios geológicos. Este estudio sirvió para caracterizar geotécnicamente los suelos de Antofagasta en cuanto a la profundidad de la roca, el grado de compactación y la composición del suelo. La Figura 1 muestra esta caracterización, la cual tiene la limitación de estar basada en prospecciones superficiales. 
Aunque la roca aflora en muchos lugares, también se presenta a gran profundidad en sectores localizados y de área reducida. El sector costero de la ciudad presenta roca a poca profundidad y los pocos suelos que se observan corresponden a rellenos artificiales de poco espesor. El casco central de la ciudad está conformado por material rocoso y grava arenosa con espesores máximos de $3 \mathrm{~m}$. En el lado centro oriente de la ciudad se observan rellenos naturales de hasta $10 \mathrm{~m}$ de espesor que corresponden a depósitos aluvionales y su composición es grava o arena limosa. En el costado nor-oriente de la ciudad se aprecian algunos terrenos conformados por arenas de baja compacidad, lo mismo ocurre en el sector sur de la ciudad, donde la compacidad de los suelos es baja, con estratos de más de $10 \mathrm{~m}$ de profundidad. Aproximadamente el $70 \%$ de la ciudad presenta características de suelo Tipo I, mientras que un $20 \%$ corresponde a suelo Tipo II. Existen sectores aislados en la zona de Avda. Argentina, en el centro sur de la ciudad, que podrían eventualmente presentar suelo Tipo III, de acuerdo a antecedentes recopilados de edificios fundados en ese sector. También se puede encontrar este tipo de suelo en el sector nor-oriente alto, caracterizado por arenas sueltas de gran espesor.

Tabla 1: Zonificación geotécnica de Antofagasta (Aguilera, 1994)

\begin{tabular}{|c|c|c|c|c|}
\hline Denominación & descripción & compacidad & $\begin{array}{c}\text { prof. de } \\
\text { la roca }\end{array}$ & $\begin{array}{c}\text { pendiente } \\
\text { superficial }\end{array}$ \\
\hline Zona I & $\begin{array}{c}\text { arenas } \\
\text { intercaladas con } \\
\text { arenas gravosas }\end{array}$ & $\begin{array}{c}\text { media } \\
\text { densa }\end{array}$ & $\begin{array}{c}\text { mayor a } \\
2 \mathrm{~m}\end{array}$ & $\begin{array}{c}\text { variable } \\
\text { entre 0 a } \\
10 \%\end{array}$ \\
\hline
\end{tabular}

\begin{tabular}{|c|c|c|c|c|}
\hline Zona II & $\begin{array}{c}\text { arenas, } \\
\text { conchuelas, } \\
\text { arenas y gravas } \\
\text { marinas }\end{array}$ & media & $\begin{array}{c}\text { menor a } \\
2 \mathrm{~m}\end{array}$ & $\begin{array}{c}\text { menor a } \\
10 \%\end{array}$ \\
\hline Zona III & $\begin{array}{c}\text { arenas gravosas } \\
\text { intercaladas } \\
\text { con capas } \\
\text { lenticulares de } \\
\text { arena }\end{array}$ & $\begin{array}{l}\text { media muy } \\
\text { densa }\end{array}$ & $\begin{array}{c}\text { mayor a } \\
2 \mathrm{~m}\end{array}$ & $\begin{array}{l}\text { variable, } \\
\text { menor a } \\
10 \%\end{array}$ \\
\hline Zona IV & arenas & densa & $\begin{array}{c}\text { mayor a } \\
2 \mathrm{~m}\end{array}$ & $\begin{array}{c}\text { menor a } \\
20 \%\end{array}$ \\
\hline Zona V & arenas gravosas & $\begin{array}{c}\text { suelta a } \\
\text { media }\end{array}$ & $\begin{array}{c}\text { mayor a } \\
2 \mathrm{~m} \\
\end{array}$ & $\begin{array}{c}\text { entre } 10 \% \\
\text { y } 30 \% \\
\end{array}$ \\
\hline Zona VI & arenas gravosas & $\begin{array}{c}\text { suelta a } \\
\text { media }\end{array}$ & $\begin{array}{c}\text { mayor a } \\
2 \mathrm{~m} \\
\end{array}$ & $\begin{array}{c}\text { entre } 10 \% \\
\text { y } 30 \% \\
\end{array}$ \\
\hline Zona VII & $\begin{array}{c}\text { arenas, } \\
\text { conchuelas, } \\
\text { arenas y gravas } \\
\text { marinas }\end{array}$ & $\begin{array}{c}\sin \\
\text { información }\end{array}$ & $\begin{array}{c}\text { menor a } \\
2 \mathrm{~m}\end{array}$ & $\begin{array}{c}\text { menor a } \\
10 \%\end{array}$ \\
\hline Zona VIII & arenas gravosas & $\begin{array}{l}\text { suelta a } \\
\text { media }\end{array}$ & $\begin{array}{c}\text { mayor a } \\
2 \mathrm{~m}\end{array}$ & $\begin{array}{c}\text { se presenta } \\
\text { en algunos } \\
\text { lugares } \\
\text { pendientes } \\
\text { mayores al } \\
30 \% \\
\end{array}$ \\
\hline Zona IX & arenas gravosas & $\begin{array}{l}\text { suelta a } \\
\text { media }\end{array}$ & $\begin{array}{c}\text { mayor a } \\
2 \mathrm{~m}\end{array}$ & $\begin{array}{l}\text { valores } \\
\text { menores } \\
\text { al } 20 \% \\
\end{array}$ \\
\hline Zona X & $\begin{array}{c}\text { rellenos } \\
\text { artificiales, } \\
\text { conchuelas, } \\
\text { arenas y gravas } \\
\text { marinas }\end{array}$ & $\stackrel{\sin }{\text { información }}$ & $\begin{array}{c}\text { menor a } \\
2 \mathrm{~m}\end{array}$ & $\begin{array}{c}\text { menor al } \\
10 \%\end{array}$ \\
\hline Zona XI & $\begin{array}{c}\text { arenas, } \\
\text { conchuelas, } \\
\text { arenas y gravas } \\
\text { marinas } \\
\end{array}$ & $\begin{array}{l}\text { media a } \\
\text { densa }\end{array}$ & $\begin{array}{c}\text { menor a } \\
2 \mathrm{~m}\end{array}$ & $\begin{array}{c}\text { menor al } \\
10 \%\end{array}$ \\
\hline Zona XII & arenas gravosas & $\begin{array}{l}\text { suelta a } \\
\text { media }\end{array}$ & $\begin{array}{c}\text { mayor a } \\
2 \mathrm{~m}\end{array}$ & $\begin{array}{c}\text { entre } 10 \% \\
\text { a } 20 \%\end{array}$ \\
\hline Zona XIII & arenas gravosas & $\begin{array}{c}\text { media a } \\
\text { muy densa }\end{array}$ & $\begin{array}{c}\text { mayor a } \\
2 \mathrm{~m} \\
\end{array}$ & $\begin{array}{c}\text { entre } 10 \% \\
\text { a } 30 \% \\
\end{array}$ \\
\hline Zona XIV & $\begin{array}{c}\text { rellenos } \\
\text { artificiales, } \\
\text { conchuelas, } \\
\text { arenas y gravas } \\
\text { marinas }\end{array}$ & $\stackrel{\sin }{\text { información }}$ & $\begin{array}{c}\text { mayor a } \\
2 \mathrm{~m}\end{array}$ & $\begin{array}{c}\text { menores a } \\
\text { un } 10 \%\end{array}$ \\
\hline Zona XV & $\begin{array}{c}\text { areno gravoso } \\
\text { aluvional } \\
\end{array}$ & $\begin{array}{c}\text { media a } \\
\text { densa }\end{array}$ & $\begin{array}{c}\text { mayor a } \\
2 \mathrm{~m}\end{array}$ & $\begin{array}{c}\text { entre } 10 \% \\
\text { a } 30 \% \\
\end{array}$ \\
\hline Zona XVI & areno gravoso & $\begin{array}{c}\text { media a } \\
\text { densa }\end{array}$ & $\begin{array}{c}\text { mayor a } \\
2 \mathrm{~m}\end{array}$ & $\begin{array}{c}\text { entre } 10 \% \\
\text { a } 20 \%\end{array}$ \\
\hline
\end{tabular}

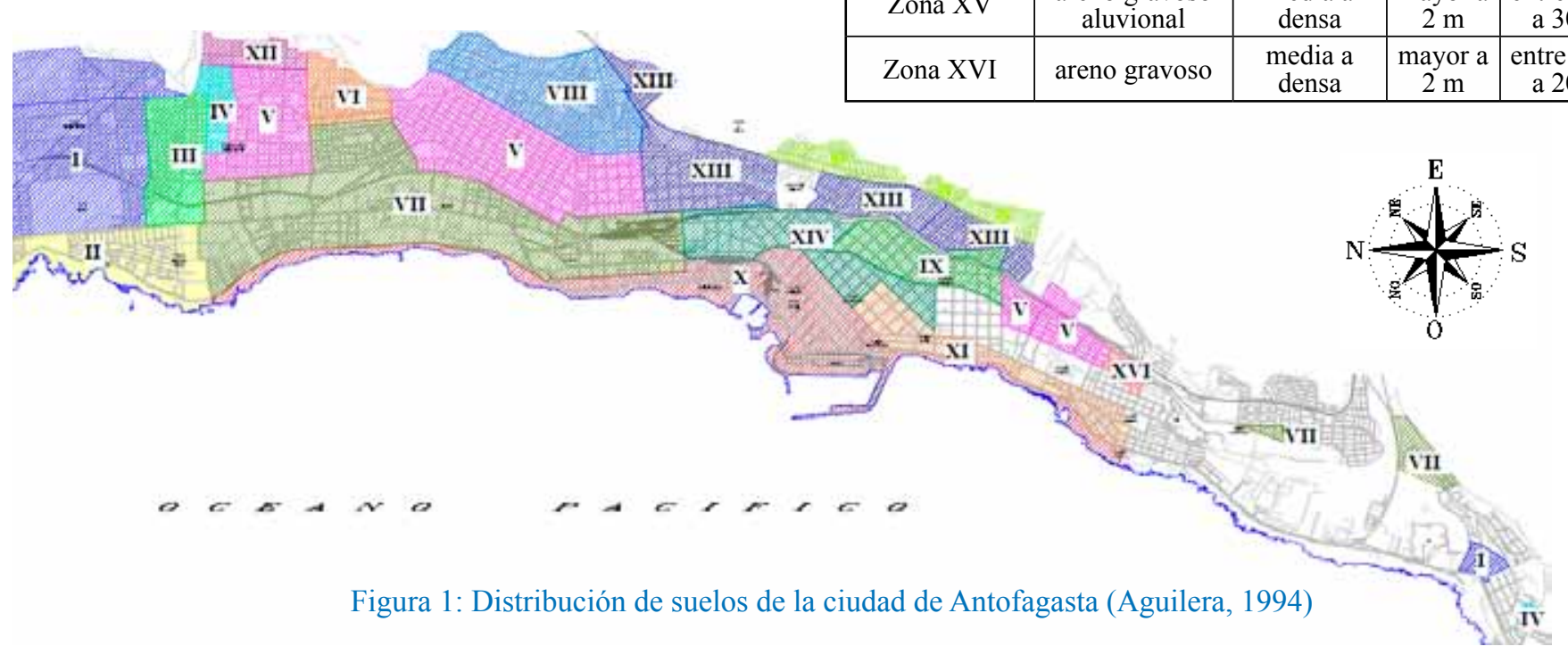




\section{La edificación en Antofagasta}

La edificación en Antofagasta se ha desarrollado a través de diferentes materiales, desde el tradicional "palo ahogado" (entramado de madera relleno con hormigón de baja calidad, reforzado con una malla de alambre), típico de las estructuras antiguas del sector centro de la ciudad, hasta modernos edificios altos construidos en base a hormigón armado. En los sectores periféricos ubicados al oriente de la ciudad predomina la construcción con material ligero y la autoconstrucción, mientras que la mayoría de la edificación baja se ha desarrollado en albañilería confinada. El 'boom' inmobiliario que se generó en la ciudad a partir del año 1995 y el alto costo del metro cuadrado de terreno ha generado la proliferación de la edificación en altura. La Figura 2 muestra la distribución de edificios por pisos en la ciudad usando una estadística de construcción hasta el año 2010. La cantidad de edificios de 10 pisos o más alcanza a 115.

La mayoría de los edificios de 4 o más pisos se ubican en la zona centro sur de la ciudad, como se indica en la Figura 3. Muy pocos edificios se ubican en la zona de mayor expansión de la ciudad como lo es el sector norte. Cabe hacer notar que el sector centro norte de la ciudad corresponde a una sector cuyo uso de suelo está destinado a la industria. De los sectores altos de la ciudad, el que presenta el mayor número de edificios altos es la población Coviefi, cuyo principal material de fundación es roca. Del catastro de edificios mostrado en la Figura 3 (obtenido de los archivos de la Ilustre Municipalidad de Antofagasta) y la distribución de suelos indicado en la Figura 1, se concluyó que el $90 \%$ de los edificios altos (10 o más pisos) de la ciudad está fundado en roca (Aguilera, 1994).

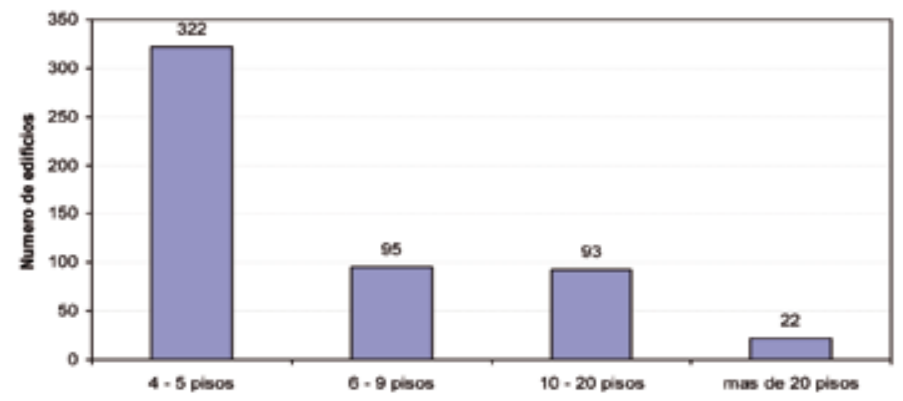

Figura 2: Distribución de edificios en base al número de pisos en Antofagasta hasta el año 2010

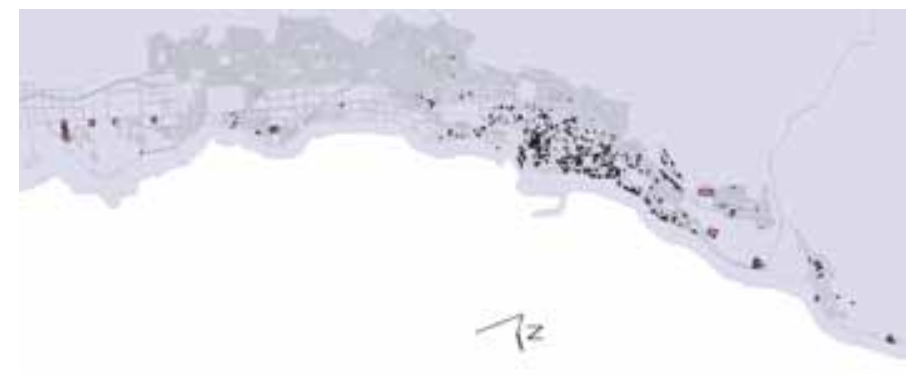

Figura 3. Ubicación geográfica de edificios de 4 o más pisos en Antofagasta hasta el año 2010

\section{Metodología de análisis}

En este estudio se analizaron nueve edificios de la ciudad de Antofagasta, la mayoría de ellos fundados en roca, excepto el edificio Pérez Zujovic el cual está fundado en arena y se muestra a modo ilustrativo en la Figura 4. Las características más importantes de los edificios, tales como año de construcción, altura, número de pisos, etc. se muestran en la Tabla 2. Un análisis detallado del perfil bio-sísmico de los edificios puede encontrarse en Ríos (2006), el cual está basado en metodología de calificación sísmica de edificios de hormigón armado propuesta por Guendelman et al. (1997). Los edificios fueron modelados usando el programa computacional ETABS determinándose para cada uno de ellos los períodos con mayor masa traslacional para dos direcciones perpendiculares y el porcentaje de masa equivalente asociado, ver Tabla 3. Con esta información se procedió a realizar un análisis modal espectral considerando primero la norma $\mathrm{NCh} 433$ Of. 96 Mod. 2009 (normativa antigua) y luego incorporando las disposiciones estipuladas en el Decreto 117. Para efectos comparativos, solo se varió el espectro de diseño, considerando la misma clasificación de suelos en ambos casos, es decir, suelo Tipo I para los edificios fundados en roca y suelo Tipo II para la Torre Pérez Zujovic fundada en arena. En este punto cabe hacer notar que la clasificación sísmica de suelos dada en la norma NCh433 Of. 96 Mod. 2009 difiere de la estipulada en el Decreto 117, por lo tanto, es probable que un suelo clasificado como Tipo II según la normativa antigua, sea clasificado ahora según la nueva normativa como Tipo III. Una comparación de los espectros de diseño para zona sísmica III se muestra en las Figuras 5 a 7, considerando distintos tipos de suelos. Los gráficos han sido realizados utilizando un factor de importancia del edificio $I=1$, una aceleración máxima 
efectiva del suelo $\mathrm{A}_{0} / \mathrm{g}=0.4$, factor de modificación de la respuesta estructural $R_{0}=11$ y un periodo con mayor masa equivalente en la dirección de análisis $\mathrm{T}^{*}=1 \mathrm{~s}$. A partir de estas comparaciones, es posible apreciar que para periodos bajos (menores a $1 \mathrm{~s}$ ), el cambio en la demanda sísmica es mínimo. Por otra parte, para períodos altos (mayores a $1 \mathrm{~s}$ ), la demanda sísmica de acuerdo a la nueva normativa puede llegar a triplicar la demanda impuesta por la $\mathrm{NCh} 433$ Of. 96 Mod. 2009, como ocurre en el caso de suelos Tipo II.

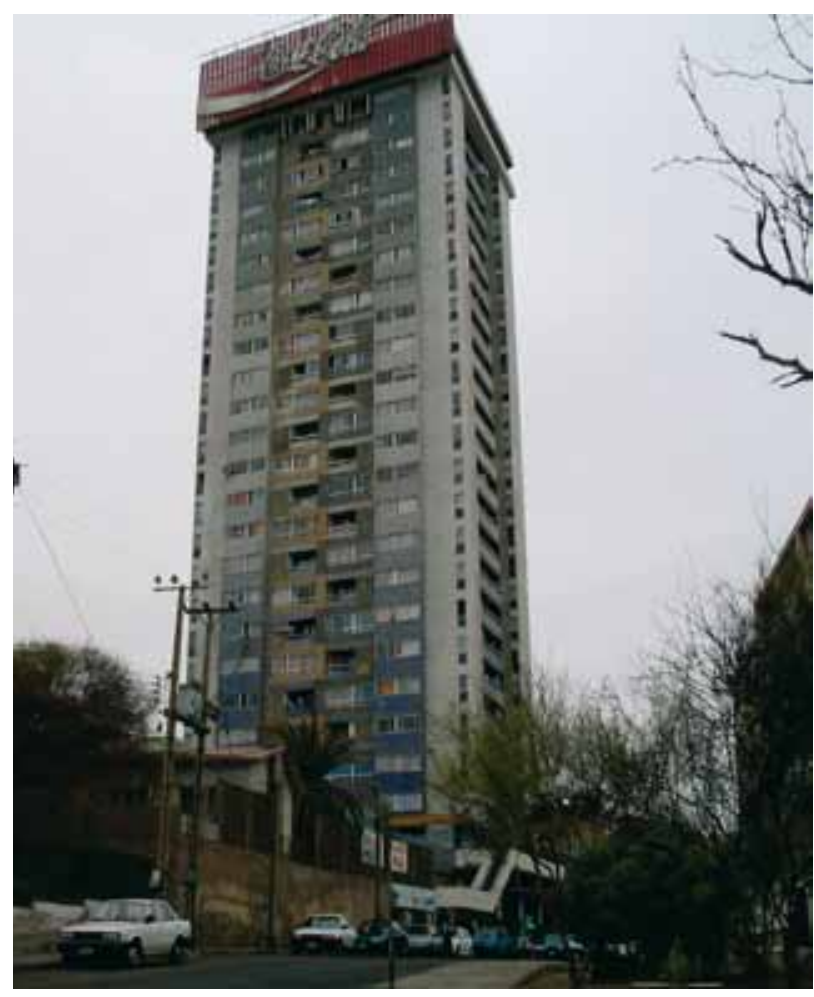

Figura 4: Torre Edmundo Pérez Zujovic

Tabla 2: Características de los edificios analizados

\begin{tabular}{|l|c|c|c|c|c|c|c|c|}
\hline \multicolumn{1}{|c|}{ Edificio } & Año & $\begin{array}{c}\text { Altura } \\
(\mathrm{m})\end{array}$ & $\begin{array}{c}\text { Area } \\
\text { Total } \\
\left(\mathrm{m}^{2}\right)\end{array}$ & Pisos & Subterr. & Niv. & \multicolumn{2}{|c|}{ Rigideces } \\
\hline Grecia & 1990 & 33,7 & 2304,6 & 12 & 1 & 13 & Regular & Irregular \\
\hline Las Palmas & 1997 & 38,6 & 6580,0 & 14 & 1 & 15 & Regular & Irregular \\
\hline Los Sauces & 1999 & 37,9 & 15214,8 & 15 & 0 & 15 & Regular & Irregular \\
\hline Mar del Sur & 1993 & 33,2 & 1613,8 & 13 & 0 & 13 & Regular & Irregular \\
\hline $\begin{array}{l}\text { Mirador del } \\
\text { Sur }\end{array}$ & 1999 & 41,4 & 6054,3 & 15 & 1 & 16 & Regular & Irregular \\
\hline $\begin{array}{l}\text { Pérez } \\
\text { Zujovic }\end{array}$ & 1978 & 74,5 & 14104,1 & 27 & 1 & 28 & Regular & Regular \\
\hline $\begin{array}{l}\text { Plaza } \\
\text { Foresta }\end{array}$ & 2002 & 46,9 & 5500,9 & 17 & 1 & 18 & Irregular & Irregular \\
\hline Terramar & 1996 & 32,3 & 2911,2 & 12 & 0 & 12 & Irregular & Irregular \\
\hline Torremarina & 1994 & 46,2 & 7364,0 & 17 & 1 & 18 & Regular & Irregular \\
\hline
\end{tabular}

Tabla 3: Resultados del análisis modal

\begin{tabular}{|l|c|c|c|c|c|c|c|}
\hline Edificio & Niveles & $\begin{array}{c}\text { Modos } \\
\text { para } \\
90 \% \\
\text { masa } \\
\text { equiv. }\end{array}$ & $\begin{array}{c}\mathrm{T}_{\mathrm{x}}{ }^{*} \\
\mathrm{~S}\end{array}$ & $\begin{array}{c}\% \text { de masa } \\
\text { equiv. X } \\
\text { asociada }\end{array}$ & $\begin{array}{c}\mathrm{T}_{\mathrm{y}}{ }^{*} \\
\mathrm{~s}\end{array}$ & $\begin{array}{c}\% \text { masa } \\
\text { equiv. Y } \\
\text { asociada }\end{array}$ & $\begin{array}{c}\text { Tipo } \\
\text { Suelo }\end{array}$ \\
\hline Grecia & 13 & 20 & 0,42 & 56,1 & 0,34 & 61,1 & Roca \\
\hline Las Palmas & 15 & 9 & 0,43 & 66,5 & 0,48 & 69,4 & Roca \\
\hline Los Sauces & 15 & 9 & 0,30 & 70,7 & 0,40 & 35,9 & Roca \\
\hline Mar del Sur & 13 & 10 & 0,61 & 61,1 & 0,40 & 66,3 & Roca \\
\hline $\begin{array}{l}\text { Mirador del } \\
\text { Sur }\end{array}$ & 16 & 9 & 0,45 & 36,7 & 0,65 & 68,6 & Roca \\
\hline $\begin{array}{l}\text { Pérez } \\
\text { Zujovic }\end{array}$ & 28 & 14 & 1,10 & 61,0 & 1,57 & 61,8 & Arena \\
\hline $\begin{array}{l}\text { Plaza } \\
\text { Foresta }\end{array}$ & 18 & 13 & 0,40 & 36,9 & 0,63 & 63,1 & Roca \\
\hline Terramar & 12 & 9 & 0,37 & 58,1 & 0,30 & 63,9 & Roca \\
\hline Torremarina & 18 & 12 & 0,71 & 46,4 & 0,59 & 67,1 & Roca \\
\hline
\end{tabular}

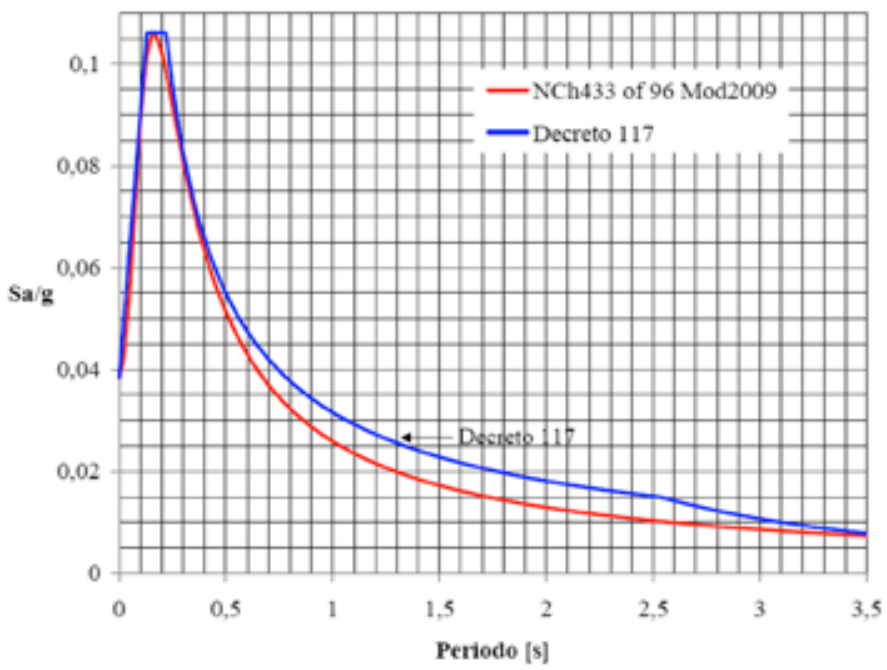

Figura 5: Comparación de espectros de diseño para zona sísmica III y suelo Tipo I, considerando $\mathrm{I}=1, \mathrm{R}_{0}=11$ y $\mathrm{T}^{*}=1 \mathrm{~s}$

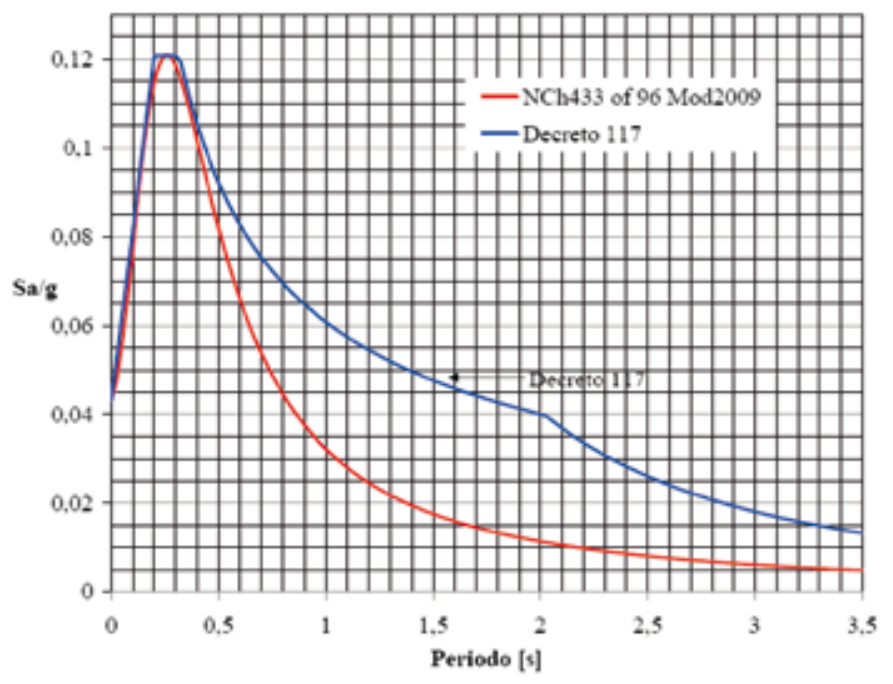

Figura 6: Comparación de espectros de diseño para zona sísmica III y suelo Tipo II, considerando I $=1, \mathrm{R}_{0}=11 \mathrm{y} \mathrm{T}^{*}=1 \mathrm{~s}$ 


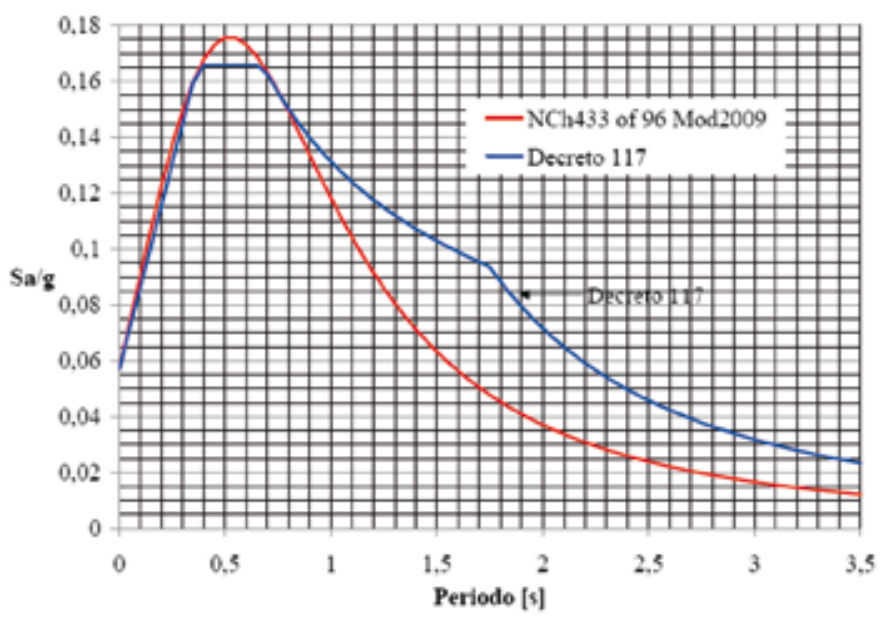

Figura 7: Comparación de espectros de diseño para zona sísmica III y suelo Tipo III, considerando I $=1, \mathrm{R}_{0}=11$ y T* $=1 \mathrm{~s}$

\section{Resultados}

En esta sección se resumen los principales resultados obtenidos para los nueve edificios analizados. La Tabla 4 muestra el incremento porcentual en cortes basales y momentos volcantes producto de las disposiciones del Decreto 117, con respecto a la NCh 433 Of. 96 Mod. 2009, y antes de considerar las disposiciones de corte basal mínimo. En el caso de edificios fundados en roca, el corte basal y momento volcante se amplifican por un factor máximo del orden de 1.1. Esto en contraste con el edificio Perez Zujovic, fundado en arena, donde por ejemplo para un sismo en la dirección Y, los factores de amplificación son del orden de 1.8 y 2.8 , para corte basal y momento volcante, respectivamente.

Tabla 4: Incrementos de cortes basales y momentos volcantes antes de considerar la condición de corte basal mínimo

\begin{tabular}{|l|c|c|c|c|}
\hline \multicolumn{5}{|c|}{ Diferencias antes de considerar $\mathrm{Q}_{\min }$} \\
\hline Edificio & Qxx, \% & Qyy, \% & My, \% & $\mathrm{Mx} \%$ \\
\hline Pérez Zujovic & 54,9 & 82,5 & 100,2 & 185,5 \\
\hline Grecia & 5,1 & 3,4 & 4,9 & 3,1 \\
\hline Las Palmas & 4,8 & 6,3 & 4,7 & 6,3 \\
\hline Mar del Sur & 8,5 & 3,8 & 10,6 & 3,9 \\
\hline Mirador del Sur & 7,0 & 9,4 & 7,4 & 11,9 \\
\hline Plaza Foresta & 3,9 & 9,7 & 3,7 & 11,5 \\
\hline Terramar & 4,0 & 3,0 & 3,4 & 2,6 \\
\hline Los Sauces & 2,5 & 3,5 & 2,5 & 3,5 \\
\hline Torremarina & 9,6 & 8,9 & 11,0 & 10,3 \\
\hline
\end{tabular}

Este escenario cambia al considerar las disposiciones de corte basal mínimo, ya que en la mayoría de los edificios los cortes basales son menores que el mínimo, aún considerando el Decreto 117, y por lo tanto al escalar las solicitaciones de corte, los cortes basales según la NCh433 Of. 96 Mod. 2009 y el Decreto 117 coinciden con el mínimo. Esto explica la nula diferencia en el corte basal en la mayoría de los edificios, ver Tabla 5 . En cuanto a los momentos volcantes, las diferencias son prácticamente nulas para los edificios fundados en suelo Tipo I, mientras que éstas se reducen considerablemente en el caso del edificio Pérez Zujovic, fundado en suelo Tipo II, con respecto a las diferencias antes de considerar el corte mínimo (Tabla 4). A pesar de esta reducción, las diferencias siguen siendo importantes, llegando al 56\% para un sismo en la dirección Y. Estas diferencias pueden ser explicadas considerando la distribución de fuerza cortante en los distintos niveles, lo cual da una idea de la importancia de cada modo en la combinación modal CQC. Para un edificio fundado en suelo Tipo I, como por ejemplo Torremarina, la diferencia en la distribución de cortes por niveles entre ambas normativas es prácticamente nula, tal como se muestra en la Figura 8. En contraste, para la torre Pérez Zujovic, ver Figura 9, la doble curvatura que presenta la distribución de cortes en altura correspondiente a la NCh433 Of. 96 Mod. 2009, indica que no solo el modo con mayor masa traslacional controla la distribución de cortes, sino que los modos siguientes con menor periodo, pero con una mayor demanda sísmica, también influyen de manera considerable en la respuesta de la estructura. Esta doble curvatura no está presente en la distribución de cortes en altura asociada a la nueva normativa, lo que indica que prácticamente el primer modo controla la respuesta de la estructura. Esto se debe al aumento en la demanda sísmica propuesto en el Decreto 117 para periodos altos en suelos Tipo II. Por ejemplo, en el caso del edificio Pérez Zujovic, con un periodo fundamental de $1.57 \mathrm{~s}$, la demanda sísmica asociada a dicho periodo se amplifica por un factor 2.8 .

Finalmente se analizan los desplazamientos del nivel superior y el máximo desplazamiento de entrepiso en el centro de masa para todos los edificios, comparándose los resultados obtenidos para el DS117 con respecto a los obtenidos para la NCh433 Of. 96 Mod. 2009. La Tabla 6 muestra dicha comparación, donde claramente se aprecian 
diferencias menores para los edificios fundados en roca. Sin embargo, dichas diferencias son importantes para la Torre Perez Zujovic, fundada en suelo Tipo II, pudiendo los desplazamientos incrementarse hasta en un $80 \%$.

Tabla 5: Incrementos de cortes basales y momentos volcantes después de considerar la condición de corte basal mínimo

\begin{tabular}{|l|c|c|c|c|}
\hline & \multicolumn{4}{|c|}{ Diferencias después de considerar $\mathrm{Q}_{\min }$} \\
\hline Edificio & Qxx, \% & \multicolumn{1}{|c|}{ Qyy, \% } & \multicolumn{1}{c|}{ My, \% } & Mx, \% \\
\hline Pérez Zujovic & 0,0 & 0,0 & 29,2 & 56,5 \\
\hline Grecia & 0,0 & 0,0 & $-0,2$ & $-0,3$ \\
\hline Las Palmas & 0,0 & 0,0 & $-0,1$ & 0,0 \\
\hline Mar del Sur & 0,0 & 0,0 & 1,9 & 0,0 \\
\hline Mirador del Sur & 0,0 & 0,0 & 0,4 & 2,3 \\
\hline Plaza Foresta & 0,0 & 0,0 & $-0,2$ & 1,6 \\
\hline Terramar & 0,0 & 3,0 & $-0,5$ & 2,6 \\
\hline Los Sauces & 2,5 & 0,0 & 2,5 & 0,0 \\
\hline Torremarina & 0,0 & 0,0 & 1,3 & 1,3 \\
\hline
\end{tabular}

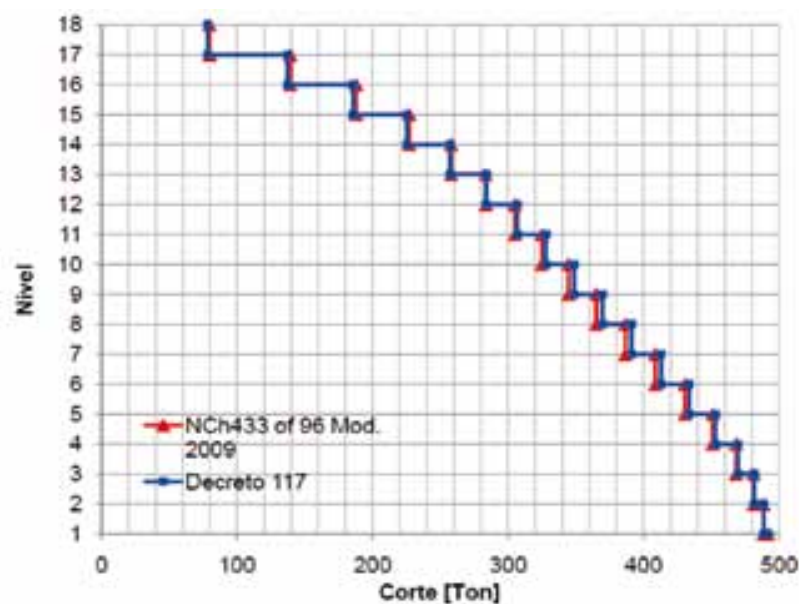

Figura 8: Distribución de la fuerza de corte a nivel de piso para el edificio Torremarina. Se ha considerado la condición de corte mínimo.

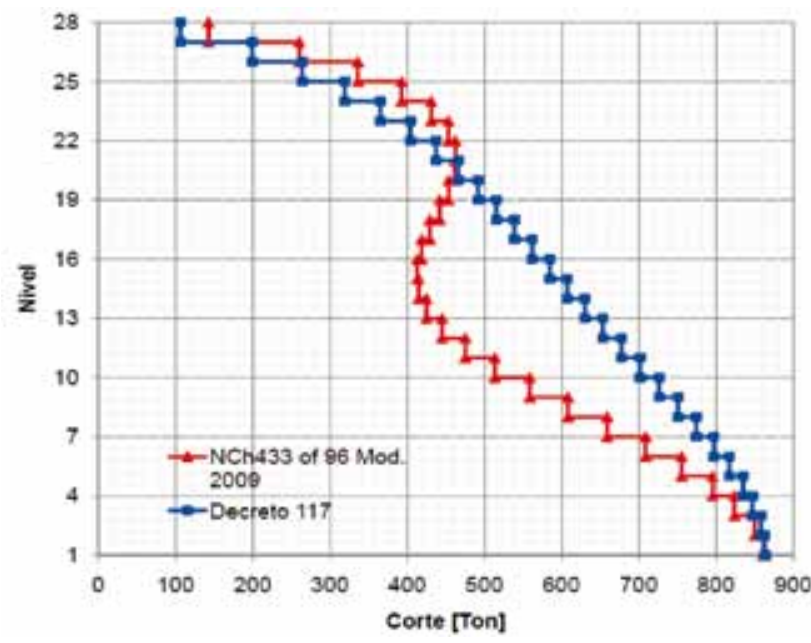

Figura 9: Distribución de la fuerza de corte a nivel de piso para el edificio Pérez Zujovic. Se ha considerado la condición de corte mínimo.
Tabla 6: Incremento de desplazamientos usando el DS117 respecto a la NCh 433 Of. 96 Mod. 2009.

\begin{tabular}{|c|c|c|c|}
\hline & \multirow{3}{*}{$\begin{array}{c}\begin{array}{c}\text { Desplazamiento de } \\
\text { nivel superior }\end{array} \\
\% \\
\text { Diferencia } \\
\end{array}$} & \multirow{3}{*}{\begin{tabular}{|c|}
$\begin{array}{c}\text { Máximo desplazamiento } \\
\text { de entrepiso en centro } \\
\text { de masa }\end{array}$ \\
$\%$ \\
Diferencia \\
\end{tabular}} \\
\hline & & & \\
\hline Edificio & & & \\
\hline $\begin{array}{l}\text { Pérez } \\
\text { Zujovic }\end{array}$ & \begin{tabular}{|l|} 
Sismo X \\
Sismo Y
\end{tabular} & $\begin{array}{l}31,9 \\
62,5\end{array}$ & $\begin{array}{l}37,7 \\
79,9\end{array}$ \\
\hline Grecia & \begin{tabular}{|l|} 
Sismo X \\
Sismo Y
\end{tabular} & $\begin{array}{l}0,0 \\
0,0\end{array}$ & $\begin{array}{c}0,0 \\
-0,6\end{array}$ \\
\hline Las Palmas & \begin{tabular}{|l|} 
Sismo X \\
Sismo Y \\
\end{tabular} & $\begin{array}{l}0,0 \\
0,0\end{array}$ & $\begin{array}{l}0,5 \\
0,9\end{array}$ \\
\hline Los Sauces & \begin{tabular}{|l|} 
Sismo X \\
Sismo Y
\end{tabular} & $\begin{array}{l}3,7 \\
0,0\end{array}$ & $\begin{array}{l}2,0 \\
0,0\end{array}$ \\
\hline Mar del Sur & \begin{tabular}{|l|} 
Sismo X \\
Sismo Y \\
\end{tabular} & $\begin{array}{l}2,8 \\
0,0 \\
\end{array}$ & $\begin{array}{l}2,8 \\
0,0 \\
\end{array}$ \\
\hline $\begin{array}{l}\text { Mirador del } \\
\text { Sur }\end{array}$ & \begin{tabular}{|l|} 
Sismo X \\
Sismo Y \\
\end{tabular} & $\begin{array}{l}2,4 \\
2,4\end{array}$ & $\begin{array}{l}2,6 \\
3,1 \\
\end{array}$ \\
\hline Plaza Foresta & \begin{tabular}{|l|} 
Sismo X \\
Sismo Y
\end{tabular} & $\begin{array}{l}0,0 \\
1,9\end{array}$ & $\begin{array}{l}0,0 \\
1,2\end{array}$ \\
\hline Terramar & \begin{tabular}{|l|} 
Sismo X \\
Sismo Y \\
\end{tabular} & $\begin{array}{l}0,0 \\
2,8\end{array}$ & $\begin{array}{l}0,0 \\
2,0\end{array}$ \\
\hline Torremarina & \begin{tabular}{|l|} 
Sismo X \\
Sismo Y
\end{tabular} & $\begin{array}{l}3,1 \\
1,7\end{array}$ & $\begin{array}{l}3,9 \\
1,7\end{array}$ \\
\hline
\end{tabular}

\section{Discusión y conclusiones}

Del presente estudio se puede concluir que para los edificios analizados y fundados en suelo Tipo I, edificios entre 12 y 18 niveles, los esfuerzos cortantes y momentos volcantes basales determinados aplicando ambas normativas, son para efectos prácticos iguales. En cuanto al edificio Pérez Zujovic fundado en suelo Tipo II, la condición de corte mínimo hace que el esfuerzo de corte basal sea igual por ambas normativas. Respecto a los momentos volcantes en cada dirección, aplicando el decreto se incrementa en $29.2 \%$ y $56.5 \%$ con respecto a lo antes vigente. Este incremento se explica debido al importante aumento en la demanda sísmica propuesto en el Decreto 117 para períodos fundamentales mayores a $1 \mathrm{~s}$ en suelos Tipo II y III, donde también se encuentran diferencias importantes en los desplazamientos.

Cabe hacer notar que de los resultados obtenidos en estos edificios, en especial, aquellos fundados en suelo Tipo I, no se puede concluir que la nueva normativa no tiene implicancia en el diseño de edificios altos, ya que si bien los resultados de la aplicación del Decreto 117 son muy similares a los de la antigua normativa, los cambios normativos afectaron tanto a la norma NCh433 Of. 96 
Mod. 2009 como a la norma de Hormigón Armado NCh430

Of. 2008. Esta última fue eliminada por el Decreto 118

(MINVU 2011b), el cual incluye cambios importantes en los requisitos de espesores de muros y elementos de borde, esfuerzo de corte último de diseño, diseño a flexión compuesta, entre otros.

Debido a que el decreto 61 de diciembre del 2011 (MINVU 2011c) deroga el decreto 117 considerado en este trabajo, se requiere por lo tanto estudiar ahora los efectos del decreto 61 en el diseño sísmico de edificios. Aparte de revisar los casos de roca y arena se necesita evaluar los casos de otros tipos de suelos de fundación, lo cual debería realizarse para edificios en otras ciudades.

\section{Referencias}

Aguilera, M. (1994). Zonificación geotécnica de la ciudad de Antofagasta. Memoria de título de Ingeniero Civil, Universidad Católica del Norte, Antofagasta

Guendelman, T., Guendelman, M. y Lindenberg, J. (1997). Perfil Bío-Sísmico de Edificios. VII Jornadas Chilenas de Sismología e Ingeniería Antisísmica, ACHISINA, La Serena
Ministerio de Vivienda y Urbanismo, MINVU (2011a). Decreto 117. Diseño sísmico de edificios. Santiago

Ministerio de Vivienda y Urbanismo, MINVU (2011b). Decreto 118. Requisitos para diseño y cálculo de hormigón armado. Santiago

Ministerio de Vivienda y Urbanismo, MINVU (2011c). Decreto 61. Reglamento que fija el diseño sísmico de edificios. Santiago

Instituto Nacional de Normalización, INN - Chile (2010). NCh433 Of. 96 Mod. 2009. Diseño sísmico de edificios, Santiago

Instituto Nacional de Normalización, INN - Chile (2008). NCh430 Of. 2008. Hormigón armado. Requisitos de diseño y cálculo. Santiago

Ríos, H. (2006). Perfil bio-sísmico de edificios representativos de la construcción en altura de la ciudad de Antofagasta. Memoria de título de Ingeniero Civil, Universidad Católica del Norte, Antofagasta 